\title{
Justice as a Service
}

\author{
Paolo Fantozzi ${ }^{1}$ (D), Luigi Laura ${ }^{2}$ (D), Antonio Nuzzo $^{3}$, and Riccardo Piselli ${ }^{3}$ \\ ${ }^{1}$ Dip. di Ingegneria informatica, automatica e gestionale Antonio Ruberti \\ Università di Roma"La Sapienza", Roma, Italy. \\ ${ }^{2}$ International Telematic University Uninettuno, Roma, Italy \\ ${ }^{3}$ Dip. di Giurisprudenza, Università Luiss Guido Carli, Roma, Italy \\ E-mail: paolo.fantozzi@uniroma1.it, \\ luigi.laura@uninettunouniversity.net, anuzzo@luiss.it, rpiselli@luiss.it
}

\begin{abstract}
In this paper we address an emerging service, called Justice as a Service (JaaS), that has been defined as an on-demand service, powered by tech, that challenges private and public companies by representing the consumer in their fight for justice/compensation based on laws, consumers' rights, and contract of carriage. Indeed, in recent years we have witnessed the birth of several JaaS companies, that are devoted to a certain type of claim such as compensation for flight delay or cancellation, or refunds for late shipping.

However, as we will discuss in the paper, we do believe that these are only the first generation of JaaS systems, i.e. the ones that solve easy problems. The advances in Natural Language Processing models such as the very recent GPT-3, together with the availability of suits data in electronic form, will soon allow the rise of more complex systems, able either to assist lawyers in the preparation of cases or to assist the end user in more involved legal issues.

In the paper, we discuss the technical and legal challenges of these new systems.
\end{abstract}

\section{Introduction}

Over the last decade, a strong wind of change has been blowing in the legal industry. Legal technologies, that is to say technologies applied to legal services, have appeared, silently starting to reshape legal services and their supply chain, to modify the internal dynamics of traditional justice systems, and to challenge our classical conception of the law $[1,2,3]$. An essential feature of such phenomenon is its horizontal nature, given that it encompasses a broad range of technologies, applications and legal services (from intelligent legal research to predictive analysis for litigation, from review of contracts and e-discovery to online courts and online dispute resolution tools) and that it has been already addressed by different governments at the international level [4].

Another important feature is design, considering that such technologies play for the most part with 'design-based regulatory techniques' (for a definition: Yeung [5]) 
in order to alter and constrain people's behaviours. This is particularly challenging for legal professionals, which do not possess a comprehensive understanding of manipulative technologies. Hence, discussions over the restructuring of the system of legal education have bloomed $[6,7,8]$, as the quests for an integration of design into the educational offering [9].

In approaching such a universe of legal tools, central is of course the concept of service, which has received recent scholarship attention as an autonomous field of research [10]. In this sense, it could be argued that legal services and more broadly justice is increasingly being thought as a service ("Justice as a Service"). Legal services in fact are first and foremost services, which share with other services the same economic, organizational and technical tension towards efficiency, productivity and quality. However, they profoundly differ with respect to the utilities they deliver: utilities that with regards to the legal services not only can be economically quantified, but present also a sort of sacral authority, associated with relevant social and public values stemming from the role of the law in every society.

This is why framing legal technologies and JaaS within the contours of Service Science requires paying particular attention to the specifics of the law and to the normative and social implications of treating it as a service. To this end, in this paper we discuss the technical and legal challenges of these new systems, trying to frame them in a Service Science perspective.

In particular, this paper is organized as follows: next section discusses related work, whilst in Section 3 we provide the current landscape of JaaS and the related, more general LegalTech services. Section 4 addresses legal issues and challenges, whilst Section 5 focuses on the tech issues, and in particular the rising NPL AI Transformer architectures, that will play a major role in the sector. Finally, Section 6 presents concluding remarks.

\section{Background Work}

We need to distinguish two different lines of related work: on the one side we have the literature related to innovation and technical aspects of legal services, and on the other we have the Service Science context.

Technical aspects of legal services. As previously mentioned, the legal services in fact are first and foremost "services", and thus there is a vast corpus of research focused on the the technical aspects of legal services, also called LawTech or LegalTech. We refer the interested reader to the recent book of Hartung, Bues, and Halbleib [11] on the topic, and mention that there are raising issues also on the educational side of these aspects $[12,6,13]$. If we focus on new tech companies, Sako et al. [14] present a comparison between the startup ecosystem of two areas (fintech and lawtech) and three locations (London, New York City, and San Francisco Bay Area).

Service Science Context. However, in this vast amount of literature, so far in the context of Service Science, short for Service Science, Management, Engineering, and Design (SSMED) $[15,16]$ the topic has been mostly unaddressed: just to be sure not to miss anything, we checked the proceedings of all the previous editions of the International Conference on Exploring Services Science searching for related work. Thus, in this paper, as we mentioned before, we try to frame this types of systems in a Service Science perspective. However, as it will be clear from the next sections, the scenario we are facing is very rich and heterogeneous, preventing us from modeling it in a more formal way, e.g., using tools such as Viable System Model [17] or providing 
a more detailed picture like the one presented by Drăgoicea et al. for the case of Public Safety as a Service [18].

\section{JaaS and LegalTech services landscape}

In recent years we have witnessed the birth of several JaaS companies, we mention some of them in the following just to provide some concrete examples:

- AirHelp is devoted to help users to claim compensation for flight delay or cancellation.

- BillFixers lowers the rates on users' bills in exchange for half of saved the first year.

- CellBreaker focus on users that want to switch mobile phone contracts, preventing them to waste money on termination fees.

- Paribus monitors your Amazon orders; if an item you bought drops its price shortly after your purchase, you are entitled to the price difference. Paribus sends the claim to Amazon and charges a fee of the refund.

- Pixsy finds and fights images used without permission.

- 71lbs is devoted to help companies getting refunds for late shipping.

As we can see from the list above, each of the company is devoted to a certain type of claim. However, we do believe that these are only the first generation of JaaS systems, i.e. the ones that solves easy problems. The advances in Natural Language Processing, discussed in more detail in Section 5, together with the availability of suits data in electronic form, will soon allow the rise of more complex systems, able either to assist lawyers in the preparation of cases or to assist the end user in more involved legal issues.

Zillmer [19] defines Justice as a Service (JaaS) as an on-demand service, powered by tech, that challenges private and public companies by representing the consumer in their fight for justice/compensation based on laws, consumers' rights, and contract of carriage.

JaaS can be framed within the more general concept of LawTech (also called LegalTech). The UK Law Society defined LawTech as broadly, technologies which aim to support, supplement or replace traditional methods for delivering legal services, or transactions; or which improve the operation of the justice system [20].

The UK Law Society also produced, in 2019, an interesting report [21] about the adoption of lawtech solutions, that provides a wealth of information; as they observe, the lawtech remains less mature than other field of digital disruption, such as fintech, where "funding and regulatory alignment are more advanced".

Anyway, despite the field seems less mature than others, it is one of the most active sectors for investors, with an overall funding between $6 \mathrm{~B}$ and $9 \mathrm{~B} \mathrm{USD}$, according to different sources ${ }^{1}$; the website legalpioneer.org addresses more than $5 \mathrm{k}$ startups in approximately 800 locations worldwide. Amongst the most funded companies, we cite Notarize (Boston, 83M USD), a cloud-based legal electronic documents management software provider, LawGeex (Tel Aviv, 45M USD), an automated legal contract analyzer, and Onna (San Francisco, 43M USD), SaaS eDiscovery (see later).

We tried to categorize the different LawTech applications in Table 1, where we distinguish also the market, i.e. business to business (B2B), business to customer $(\mathrm{B} 2 \mathrm{C})$, or in-house solutions, i.e. systems that are used within an organization or

\footnotetext{
${ }^{1}$ See www.tracx.com and www.legalcomplex.com
} 
Table 1. Different types of LawTech applications and their markets

\begin{tabular}{l|c|c|c|} 
& B2B & in-house & B2C \\
\hline Case and Workflow Management & $\checkmark$ & $\checkmark$ & \\
Practice and Dossier Management & $\checkmark$ & $\checkmark$ & \\
Document and Contract Automation & $\checkmark$ & $\checkmark$ & $\checkmark$ \\
Legal Research (database and tools) & $\checkmark$ & $\checkmark$ & \\
Legal Document templates and generation & $\checkmark$ & $\checkmark$ & $\checkmark$ \\
eDiscovery & $\checkmark$ & $\checkmark$ & \\
GDPR privacy & $\checkmark$ & & $\checkmark$ \\
Debt Collection & $\checkmark$ & $\checkmark$ & \\
Marketplaces & $\checkmark$ & & $\checkmark$ \\
Chatbots (Virtual Legal Assistant) & & & $\checkmark$ \\
Virtual Data Room (VDR) & $\checkmark$ & & \\
Contract Management & $\checkmark$ & $\checkmark$ & $\checkmark$ \\
Document Management and Capture & $\checkmark$ & $\checkmark$ & $\checkmark$ \\
Legal BIM (Building Information Management) & $\checkmark$ & $\checkmark$ & $\checkmark$ \\
\hline
\end{tabular}

a company. Many of the types listed in the table should be self evident; we might need to clarify that eDiscovery, according to Oard and Webber [22], is the process by which one party is entitled to "discover" evidence in the form of "electronically stored information" that is held by another party and that is relevant to some matter that is the subject of civil litigation(i.e., what is commonly called a "lawsuit").

It is also interesting to mention the legal BIM applications, i.e. the legal aspects of the Building Information Management (see the work of Drăgoicea et al. [23] for a Service Science perspective of intelligent building management).

\section{Legal issues and challenges of JaaS}

\subsection{JaaS's impact on the law and its positive outcomes}

Legal technologies and JaaS seem to impact on the law in three different ways: in particular, they influence the production of the law, they change people's understanding of the law, and they import disruptive changes in the way legal services are delivered [24]. With regard to the production of law, some have argued that emerging technologies will lead to the death of rules and standards, leaving the pace to a new form of law called micro-directive [25]. Individuals' understanding of the law would be facilitated as well: cutting-edge information technologies are increasingly allowing people to know instantaneously every happened change in the law, making as a result the law less obscure and more intelligible. Finally, as machines are already taking on many tasks historically managed by traditional professions [26], there are good reasons for believing that the legal industry will make no exception [3]. This would foster the "better for less" imperative that has come to characterize the legal profession. 
JaaS undoubtedly holds a radiant future. Legal technologies, if correctly framed in a comprehensive matrix of resources, processes and people, are an invaluable resource for legal professionals, clients and people in general.

Firstly, JaaS guarantees a more generalised access to justice, making the service more affordable, cost-effective, easier to use, and available to many more people. This would result in an overall enhancing of the rule of law and a better access to justice [27]. The point is crucial considering that according to the Oecd millions of people live in countries where the rule of law is little more than a pipe dream. JaaS, from this perspective, could serve as an instrument of collective and social action rather than a tool that would foster inequalities in the access to services [28]. The fear that a 'massification' of legal services would reduce their overall quality enhancing disparities between social classes seems misplaced, given that even now inequalities exist and, in most cases, people do not have access to social justice because they ignore their rights. Instead, bringing legal services in the hands of individuals through userfriendly applications and devices would probably facilitate the creation of a real and widespread legal culture, which in turn would result in a better justice for everyone, especially in developing countries.

Secondly, it makes the law and justice more standardised, uniformed, and in a sense more global. The legal industry has always been said to be "immune" to the globalisation of markets. It could be said that it is the very nature of the law, which is essentially a by-product of a complex socio-cultural matrix, that escapes from external and endogenous colonisations. However, legal technologies seem to overcome the differences between jurisdictions and to bring several positive outcomes to legal systems. In this sense, it was argued as legal tech could improve transparency, help disseminating legal information, and provide access to court decisions [29].

\subsection{The dark side of JaaS and its criticisms}

However, even the drawbacks of JaaS should not be underestimated.

Firstly, some have investigated the structural differences between science and the law [30]. What was highlighted from different angles is that the law emanating from a machine, i.e. judgements pronounced with the help of Digital, (in the form, for example, of an advices given by automatic decision-making systems to the legislative or judiciary bodies or of an agreement automatically enforced) is outside the democratic circuit, it lacks democratic legitimacy [31], and it is inherently deterministic, giving rise to a sort of new techno-normativity [32] or 'lex informatica' [33]. The law, instead, must be understood as a form of "discursive communication" [34], which is created within the political circuit by legitimate constitutional bodies or in the process of reciprocal confrontation among two negotiating parties. In the case of JaaS no discursive communication remains and the entire meaning of the law happens to be reduced to an opt-in/opt-put choice in the hands of the parties. And this could undermine the very philosophical foundations of the law, condemning in the end the entire humankind to a condition of substantial irrelevance.

A second important criticism come from those who have argued how algorithms are a sort of black-box [35], known only to some extent to their programmers. Such an opacity is quite likely to become an instrument of power and control in the hands of the tech industry, risking to jeopardize the very tenets of the rule of law. This aspect assumes particular relevance in the case of algorithmic sentencing. On the matter, some have argued that two elements might undermine the ideal of a fair trial when algorithms are involved: on the one hand, the judiciary might end up relying 
Table 2. Key points from this section: JaaS' Pros and Cons.

\begin{tabular}{|l|l|}
\hline PROs & CONs \\
\hline $\begin{array}{l}\text { - JaaS guarantees a more gener- } \\
\text { alised access to justice }\end{array}$ & JaaS lacks democratic legitimacy \\
- JaaS has the potential to im- \\
$\begin{array}{l}\text { prove fairness and equality } \\
\text { - Jaas makes law and justice more } \\
\text { standardised and, in some sense, } \\
\text { more global }\end{array}$ & $\begin{array}{l}\text { Algorithms sometimes are black- } \\
\text { box }\end{array}$ \\
\hline
\end{tabular}

on the superiority of quantitative methods over verbal reasoning (hence reducing the decision-making activity to a mere applications of quantitative data); on the other, when algorithms are kept secret it is impossible or difficult to challenge key aspects of it. The possibility to know the reasons behind a certain decision in fact is the precondition for a contestation of such decision in a contradictory procedure [36]: as a result, algorithmic opacity is very likely to compromise the right of defence in criminal and civil litigations.

Algorithmic sentencing or dispute resolution has received further criticisms on the grounds of the legal reasoning. Generally, an expert system which confront with legal issues is based on at least two components: a knowledge base and an inference engine (i.e. a pattern of reasoning which is followed to come to a conclusion). Accordingly, a debate has sparked over the problem of determining what should be included in the knowledge base [37] and the capacity of a software to reason inductively or by analogy and to give non rule-based judgements [38].

A further point regards eventually the cognitive biases that could characterize the use of standardized tools in the delivery of legal services. The issue is well illustrated by Cathy O'Neil, who argued how the problem with algorithmic decision-making lies not with the algorithms per se but with the data and information used as a knowledge base to drive inferences [39]. Such data, in fact, always mirror cognitive assumptions and biases of those who collect them and this opens the door to dangerous discriminations and ethical considerations.

\section{Technical issues and challenges of JaaS}

In this section we discuss some of the technical issues and challenges of the JaaS systems. The key technologies are, undoubtedly, Cloud, Blockchain, and Artificial Intelligence. All these technologies have been deeply studied in the context of Service Science: see, e.g., Cloud [40], Blockchain [41], and Artificial Intelligence [42, 43]; we will briefly discuss the first two, i.e. Cloud and Blockchain, and focus on the very recent progress of Natural Language Processing (NLP), the subfield of Artificial Intelligence devoted to the natural language; this subfield is the crucial key technology of JaaS since, using the words of Dale[44], the law has language at its heart.

\subsection{Cloud}

Cloud computing seems the key ingredient of the JaaS systems, providing a basic infrastructure that has several advantages, including: 
- No need of hardware: startup and companies do not need to own and manage their personal physical computing center.

- Scalability: companies can can add or subtract resources based on their needs; the system can be configured to add resources based on its usage.

- Resiliency: the system can be deployed with several degree of resiliency.

- Security: top cloud providers usually guarantee better security than self-hosted resources.

Indeed, today the cloud is undoubtedly a mature technology and summing up, it seems difficult to think, nowadays, to deploy a JaaS system that is not cloud based for the above mentioned reasons.

\subsection{Blockchain}

Blockchains are digital ledgers implemented in a distributed fashion (i.e., without a central repository) and usually without a central authority (i.e., a bank, company, or government) [45]; the blockchain technology has seen a huge popularity in recent years due to the success of Bitcoins and, later, other cryptocurrencies.

Despite being a very young technology, it can be considered very mature; there are several implementations that can be used almost immediately, including Ethereum [46] and AlgoRand [47]. However, in many JaaS systems there is a natural central authority, and thus the use of a blockchain might be limited to two cases: i) some marketing issues, i.e. when the company wants to ride the blockchain hype, or ii) an easy way of building a system that has some degrees of security.

Indeed, the potential of blockchain usage in JaaS relies mainly in its decentralized approach, that makes the blockchain a natural solution if the goal is a system shared by peers; in the context of JaaS, also the smart contracts have the potential to be, in some cases, a key ingredient of the system; smart contracts are self-enforceable and self-executable computerized contracts that are, however, already being questioned from a legal point of view [48].

\subsection{Artificial Intelligence}

As mentioned above, in this section we focus on the very recent progress of Natural Language Processing (NLP), the subfield of Artificial Intelligence devoted to the natural language.

Table 3. Key points from this section: JaaS' technologies issues.

\begin{tabular}{|l|l|}
\hline Technology & Maturity and issues \\
\hline - Cloud & $\begin{array}{l}\text { The most mature. It seems difficult to think a JaaS system } \\
\text { not using Cloud resources. }\end{array}$ \\
- Blockchain & $\begin{array}{l}\text { Can be considered a mature technology but only few JaaS } \\
\text { systems will be effectively using it. }\end{array}$ \\
- Artificial & $\begin{array}{l}\text { Despite the huge progresses of the last few years, it still can be } \\
\text { considered a non yet mature technology; it has the potential } \\
\text { to be the game changer for many JaaS system }\end{array}$ \\
\hline
\end{tabular}


The NLP area, together with other subfield of Artificial Intelligence, has been deeply impacted by the raise of Deep Learning, i.e. Artificial Neural Networks with several layers; in particular, since the introduction of the Transformer architecture in [49], the size of the natural language models seems to increase at least twice a year, with an unprecedented effectiveness. From the first implementations of these architectures, each actor in the field tried to expand the capabilities of the models increasing their size more and more. The first model to start the increasing trend was GPT [50] with 110M parameters, then Google released BERT [51] followed, 4 months later, by Open-AI GPT-2, presented in [52] with 1.5B of parameters. Six months later NVIDIA joined to this field of research presenting MegatronML in [53] with 8.3B parameters, and after more six months also Microsoft presented a transformerbased model in [54] with $17 \mathrm{~B}$ parameters. Actually the largest model is Open-AI GPT-3, presented in [55] and containing 175B parameters.

The ease of use and the effectiveness of these new NLP systems is going to completely change the current scenario. We provide just a single example, posted by Michael Tefula on Twitter ${ }^{2}$. He trained GPT-3, using only the two couple of sentences reported in Table 4, to "translate" from legal jargon to plain English. For non-technical reader without an expertise on AI, with "train" we mean that he simply showed the two couple of sentences to GPT-3, just to explain to this NLP model the task it was supposed to solve.

In Table 5 we show the results, i.e. we show three sentences in legal jargon that have been translated into plain English by GPT-3. The results are impressive; we do still have to explore and build systems out of these NLP models, and there is a huge hype around them, as also discussed in the work of Dale [56], but there is no doubt that GPT-3 and its brothers and sisters will play a major role in the development of JaaS systems; note that, however, all the risks, threats, promises, obvious solutions/results - concerning Open-AI GPT-3 and other AIs - are very important but they belong to the starting step of exploration of sustainable and resilient services: they are not

${ }^{2}$ https://twitter.com/michaeltefula/status/1285505897108832257

Table 4. The only two examples in the training data for the GPT-3 AI Model.

Legal clause: The Company and the Founders will provide the Investors with customary representations and warranties examples of which are set out in Appendix 4 and the Founders will provide the Investors with customary non-competition, non-solicitation and confidentiality undertakings.

Plain English: The startup and its founders will provide the usual assurances and guarantees on facts about the business. The founders will also agree not to work for competitors, poach employees or customers when they leave the startup, and respect confidentiality.

Legal clause: In the event of an initial public offering of the Company's shares on a US stock exchange the Investors shall be entitled to registration rights customary in transactions of this type (including two demand rights and unlimited shelf and piggy-back rights), with the expenses paid by the Company.

Plain English: If the Company does an IPO in the USA, investors have the usual rights to include their shares in the public offering and the costs of doing this will be covered by the Company. 
Table 5. The translation from legal clauses to plain English made by the GPT-3 AI Model trained on the two sentences shown in Table 4.

Legal clause: Upon liquidation of the Company, the Series A Shareholders
will receive in preference to all other shareholders an amount in respect of each
Series A Share equal to one times the Original Issue Price (the "Liquidation
Preference"), plus all accrued but unpaid dividends. To the extent that the
Company has assets remaining after the distribution of that amount, the
Series A Shareholders will participate with the holders of Ordinary Shares
pro rata to the number of shares held on an as converted basis.
Plain English: If the startup is wound up, the Series A investors will be
paid back at least what they invested and they will also share any leftover
assets with ordinary shareholders.
Legal clause: Sale of all or substantially all of the assets of the Company or a
sale of shares involving a change in control (each, a "Corporate Transaction")
will be treated in the same way as a liquidation and the proceeds of sale will
be distributed as set out in paragraph 3 . If the holders of Series A Shares
have received any Special Dividend it shall be set off against their Liquidation
Preference.
Plain English: If the company is sold, or a new owner takes control, the
proceeds of the sale will be distributed as in the liquidation clause above.
Any special dividend paid will be treated as an initial payment towards the
Series A investors.
Legal clause: If holders of at least $51 \%$ of the Series A Shares and Ordinary
Shares agree to sell their shares, there will be drag along rights so that all
remaining shareholders and option holders will be required to sell on the
same terms, provided that the dragged shareholders will not be required to
provide to the purchaser any representations or warranties except as to title
or to agree to any other terms.
Plain English: If a majority of investors agree to sell their shares, any other
investors who don't agree to sell will be forced to do so. They won't have to
provide any new assurances or guarantees but they will have to accept the
same price and terms as the others.

absolute results by themselves. Indeed, despite its huge progresses of recent years, the AI technology is not yet mature in the context of JaaS systems.

\section{Conclusions}

In this paper we provided an exploratory overview of the emerging systems providing Justice as a Service: the scenario is rich and heterogeneous, and there are a lot of challenges, both from the technical and the legal sides.

From the Service Science perspective there are, as always, more questions than answers: how to design this powerful systems? How to build multidisciplinary services to be used effectively? We do believe that the formal tools of Service Science will be effective to model and study sub-fields of JaaS, such as the ones listed in Table 1.

Anyway, viewing JaaS as a system cannot underestimate the complex role of the law in modern societies. This requires us to to reflect on how the push towards value maximization and the quality of the service might conflict with other social 
and public goals. The law, in our view, cannot be viewed as a simple resource or information shared but it is the result of the multiple interactions between all the involved parties. This particular positioning requires us to devote particular attention to the design of infrastructures and their (intentional or unintentional) effects on the multiple interactions between entities and resources exchanged.

\section{References}

[1] T. Skjolsvik and J. K. Breunig. Virtual Law Firms: An Exploration of the Media Coverage of an Emerging Archetype. Int. J. of Law and Tech. 26(1), 64-88 (2018)

[2] J. McGinnis and R. Pearce. The Great Disruption: How Machine Intelligence Will Transform the Role of Lawyers in the Delivery of Legal Services. Fordham Law Review 82(6), 3014-3065 (2014)

[3] R. Susskind. The End of Lawyers? Rethinking the Nature of Legal Services. (Oxford University Press, 2010)

[4] R. Wang. Legal technology in contemporary USA and China. Comput. Law and Sec. Review 39, 105459 (2020)

[5] K. Yeung. Are Human Biomedical Interventions Legitimate Regulatory Policy Instruments? The Oxford Handbook of Law, Regulation and Technology. (Oxford University Press, 2017)

[6] F. Ryan. Rage against the machine? Incorporating legal tech into legal education. Law Teach. online (2020)

[7] M. Smith. Integrating technology in contemporary legal education. Law Teach. 54(2), 209-221 (2020)

[8] C. Strevens, C. Welch, and R. Welch. On-line legal services and the changing legal market: Preparing law undergraduates for the future. Law Teach. 45(3), 328-347 (2011)

[9] D. Jackson. Human-Centered legal tech: Integrating design in legal education. Law Teach. 50(1), 82-97 (2016)

[10] P. P. Maglio and J. Spohrer. Fundamentals of service science. J. Acad. Mark. Sci. 36(1), 18-20 (2008)

[11] M. Hartung, M.-M. Bues, and G. Halbleib. Legal tech. (CH Beck, 2017)

[12] K. Galloway et al. The Legal Academy's Engagements with Lawtech: Technology Narratives and Archetypes as Drivers of Change. Law, Tech. \& Hum. 1, 27 (2019)

[13] V. Janeček, R. Williams, and E. Keep. Education for the provision of technologically enhanced legal services. Comput. Law and Sec. Review 40, 105519 (2021)

[14] M. Sako et al. Scaling Up Firms in Entrepreneurial Ecosystems: Fintech and Lawtech Ecosystems Compared. Available at SSRN 3520533 (2020)

[15] J. Spohrer and S. K. Kwan. Service Science, Management, Engineering, and Design (SSMED): An Emerging Discipline - Outline \& References. Int. J. of Inf. Syst. in the Serv. Sect. (IJISSS) 1(3), 1-31 (2009). 
[16] J. Spohrer, P.P. Maglio, Toward a Science of Service Systems, in Handbook of Service Science, P.P. Maglio, C.A. Kieliszewski, J.C. Spohrer (Eds.) (Springer US, Boston, MA, 2010) 157-194

[17] L. Carrubbo, M. Dragoicea, X. Hisa, A. Megaro, B. Zenelay, Is there a relationship of interdependence between resilience, viability and competitiveness? Ditron Ltd. case-study, in Proceedings of International Conference on Exploring Service Science, IESS2020, LNBIP 377, 363-376 (2020)

[18] M. Drăgoicea, N. G. Badr, J Falcão e Cunha, V. E. Oltean. From Data to Service Intelligence: Exploring Public Safety as a Service. In: Proceedings of International Conference on Exploring Service Science, IESS2018, LNBIP 331, 344-357 (Springer, 2018)

[19] H. Zillmer. Justice as a Service. https://henrikzillmer.com/justice-as-a-service (2016)

[20] The UK Law Society. Introduction to LawTech. Available at www.lawsociety.org. uk (2019)

[21] The UK Law Society. LawTech Adoption Research. Available at www.lawsociety. org.uk (2019)

[22] D. W. Oard and W. Webber. Information retrieval for e-discovery. Inf. Retr. J. 7(2-3), 99-237 (2013)

[23] M. Drăgoicea, L. Bucur, and M. Pătraşcu. A service oriented simulation architecture for intelligent building management. In: Proceedings of International Conference on Exploring Service Science, IESS2013, LNBIP 143, 14-28 (Springer, 2013).

[24] B. Alarie, A. Niblett, and A. Yoon. Law in the Future. Univ Tor Law J 66(4), 423-428 (2016)

[25] A. Casey and A. Niblett. Self-Driving Laws. Univ Tor Law J 66(4), 429-442 (2016)

[26] R. Susskind and D. Susskind. The Future of Professions. (Oxford University Press, 2015)

[27] R. Susskind. Online Courts and the Future of Justice. (Oxford University Press, 2019)

[28] P. Gowder. Transformative Legal Technology and the Rule of Law. Univ Tor Law J 68(82) 1-16 (2018)

[29] E. De of City. Janus-Faced Justice? The Role of Legal Technology in the Provision of Access to Justice. Legal Inf Manage 19, 63-65 (2019)

[30] S. Jasanoff. Just evidence: The Limits of Science in the Legal Process. J. of Law, Med. and Ethics 34, 328-341 (2006)

[31] C. B. Graber. Internet Creativity, Communicative Freedom and a Constitutional Rights Theory Response to 'Code is Law'. Transnational Culture in the Internet Age. (Edward Elgar, 2012) 
[32] M. Hildebrandt. Law as computation in the era of artificial intelligence: Speaking to the power of statistics. Univ Tor Law J 68(1), 12-35 (2018)

[33] J. R. Reidenberg. Lex Informatica: The Formulation of Information Policy Rules through Technology. Texas Law Review 76(3), 552-593 (1997)

[34] N. Luhmann. Law as a Social System. (Oxford University Press, 2008)

[35] F. Pasquale. The Black Box Society. (Harvard University Press, 2015)

[36] M. Hildebrandt. Law as Information in the Era of Data Driven Agency. Modern Law Review 79(1), 1-30 (2016)

[37] R. Susskind. Expert Systems in Law. (Oxford University Press, 1987)

[38] J. Zeleznikow. Building Decision Support Systems in Discretionary Legal Domains. Int. Review of Law, Comp. \& Tech. 14(3), 341-356 (2000)

[39] C. O'Neil. Weapons of Math Destruction. How Big Data increases Inequality and Threatens Democracy. (Crown Publishing, 2016)

[40] H. Katzan Jr. On an ontological view of cloud computing. J. of Serv. Sci. 3(1), 1-6 (2010).

[41] S. Seebacher and R. Schüritz. Blockchain Technology as an Enabler of Service Systems: A Structured Literature Review. In: Proceedings of International Conference on Exploring Service Science, IESS2017, LNBIP 279, 12-23, (Springer, 2017)

[42] P. Ferreira, J. G. Teixeira, and L. F. Teixeira. Understanding the Impact of Artificial Intelligence on Services. In: Proceedings of International Conference on Exploring Service Science, IESS2020, LNBIP 377, 202-213, (Springer , 2020)

[43] J. Reis, P. E. Santo, and N. Melão. Artificial Intelligence Theory in Service Management. In: Proceedings of International Conference on Exploring Service Science, IESS2020, LNBIP 377, 137-149, (Springer, 2020)

[44] R. Dale. Law and Word Order: NLP in Legal Tech. Nat. Lang. Eng. 25(1), 211-217, (Cambridge University Press, 2019)

[45] D. Yaga, P. Mell, N. Roby, K. Scarfone. "Blockchain technology overview". ArXiv preprint 1906.110\%8, https://arxiv.org/abs/1906.11078 (2019)

[46] C. Dannen. Introducing Ethereum and solidity. (Springer, 2017)

[47] J. Chen and S. Micali. Algorand: A secure and efficient distributed ledger. Theor Comput Sci 777, 155-183, (2019)

[48] D. Drummer and D. Neumann. Is code law? Current legal and technical adoption issues and remedies for blockchain-enabled smart contracts. J. Inf. Technol. Impact 35(4), 337-360, (2020)

[49] A. Vaswani et al. Attention is All you Need. In: Proc. of Annual Conference on Neural Information Processing Systems 2017, 5998-6008, (2017) 
[50] A. Radford et al. Improving language understanding by generative pre-training. https://www.cs.ubc.ca/ amuham01/LING530/papers/radford2018improving. pdf (Preprint, 2018)

[51] J. Devlin et al. BERT: Pre-training of Deep Bidirectional Transformers for Language Understanding. In: Proceedings of NAACL-HLT, 4171-4186, (2019)

[52] A. Radford et al. Language models are unsupervised multitask learners. Technical Report, OpenAI (2019)

[53] M. Shoeybi et al. Megatron-lm: Training multi-billion parameter language models using gpu model parallelism. ArXiv preprint 1909.08053, (2019)

[54] Microsoft Inc. Turing-NLG: A 17-billion-parameter language model by Microsoft. Microsoft Research Report, (2020).

[55] T. B. Brown et al. "Language models are few-shot learners". ArXiv preprint 2005.14165, https://arxiv.org/abs/2005.14165 (2020)

[56] R. Dale. GPT-3: What's it good for? Nat. Lang. Eng. 27(1), 113-118, (Cambridge University Press, 2021) 